\title{
$3 \quad$ Habitable Zones in Extrasolar Planetary Systems
}

Siegfried Franck, Werner von Bloh, Christine Bounama, Matthias Steffen, Detlef Schönberner and Hans-Joachim Schellnhuber

If we ask the question about the possible existence of the life outside the Earth, we first have to determine the habitable zone (HZ) for our solar system. The HZ of distances between a main sequence star and an Earth-like planet is roughly defined as the range of mean orbital radii which imply moderate planetary surface temperatures suitable for the development and subsistence of carbon-based life. The latter precondition is usually taken as the requirement that liquid water is permanently available at the planet's surface. The HZ concept was introduced by Huang [1, 2] and extended by Dole [3] and Shklovskii and Sagan [4].

For our purposes, an Earth-like planet is one similar in mass and composition to Earth. It's mass has to be sufficient to maintain plate tectonics in order for the global carbon cycle to operate and stabilize the surface temperature.

It is generally accepted that the Earth's climate is mainly determined by the atmospheric $\mathrm{CO}_{2}$ level. On geological time scales, i.e. over hundreds and thousands of million years, the Earth's climate is stabilized against increasing insolation by a negative feedback provided by the global carbon cycle: higher surface temperatures increase the precipitation and so increase the weathering rates resulting in decreasing atmospheric $\mathrm{CO}_{2}$ content and decreasing greenhouse effect. In the case of lower surface temperatures, the negative feedback loop acts analogously.

We know that at present only our Earth has liquid water at its surface. It is wellknown that Venus is much too hot for the existence of liquid water. At the Venusian orbit the insolation is too strong that the above described negative feedback breaks down: on Venus the atmosphere became so full of water vapor that no infrared radiation from the surface was able to escape to space. The resulting higher surface temperatures forced the vaporization of water to the atmosphere. This positive feedback effect is called "runaway greenhouse". On the other hand, the negative feedback loop stabilizing Earth's climate may also fail, if we would shift the planet too much away from the Sun. At such distances $\mathrm{CO}_{2}$ condenses to form $\mathrm{CO}_{2}$ clouds that increase the planetary albedo, i.e. the reflection of solar radiation, and cause lower surface temperatures. If the planet's surface would be covered with snow and ice, the albedo would increase further. This positive feedback loop is called "runaway glaciation".

Concerning Mars, we presently know of no life, but there is an ongoing discussion about the possibility that life might have been there in the past. The present Martian surface temperature is so low that $\mathrm{CO}_{2}$ condenses and the polar ice caps contain a mixture of $\mathrm{CO}_{2}$ ice and water ice (see Chap. 6, Jaumann et al.). However, the climate on Mars may not always have been so inhabitable. Early in its history, the climate is thought to have been more suitable for the existence of liquid water at or near the 
surface. The evidence comes from the interpretation of images that show the geology of the surface features (see e.g., [5]). According to our investigation of the HZ for the solar system [6], the Martian orbit position was within the HZ up to about 500 million years ago.

Jovian-type planets do not have a solid or liquid surface, covered by an atmosphere, near which organisms may exist. Therefore, usually they are considered as inhabitable. But there is the possibility that moons of giant planets are within a habitable zone. The best candidate for producing a habitable environment is Europa, the second Galilean satellite of Jupiter, with a mean density of about $3 \mathrm{~g}^{-3} \mathrm{~cm}^{-3}$ and therefore mostly composed of rock, but there are also enough volatiles. Due to low surface temperatures and additional internal heat sources (tidal heating), only a subsurface ocean could exist. A good analogue for possible life underneath the ice of Europa can be found in Lake Vostok, Antarctica. There, microorganisms exist at ice depth of $3 \mathrm{~km}$. NASA plans to investigate the possible subsurface ocean of Europa with the help of a spacecraft. Another interesting object is Saturn's moon Titan with a methane-rich atmosphere, in which photochemical reactions may create organic molecules. Titan's atmosphere will also be studied by the mission CASSINI (see Chap. 24, Foing). A detailed investigation about habitability of moons around giant planets is given by Williams et al. [7].

The same type of stability calculations described above for the solar system with the Sun as the central star can also be performed for stars other than our Sun. Such investigations are of special importance, because we now have novel techniques for the detection of extrasolar planetary systems (see also Chap. 2, Udry and Mayor). The expected basic results for the $\mathrm{HZ}$ around other central stars are relatively simple: to have a surface temperature in the range similar to the Earth's, a planet orbiting a central star

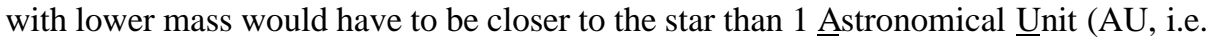
mean distance between Earth and Sun), whereas a planet orbiting a brighter star that has more mass than our Sun, would have to be farther than 1 AU from the star. But the problem is a little bit more complicated: we also have to take into account the different times that stars spend on the so-called main sequence. The main sequence is a band running from the upper left to the lower right on a plot of luminosity versus effective radiating temperature. Such a plot is called Hertzsprung-Russell diagram. Stars on the main sequence receive their energy mainly from hydrogen burning, i.e. the fusion of hydrogen to helium. In our investigation of extrasolar planetary systems [8], we have used a parameterization for the luminosity of main sequence central stars in the mass range between $20 \%$ and $250 \%$ solar masses.

Dole's [3] estimations of the HZ have been based on an optical thin atmosphere and a fixed albedo model. He finds $0.725 \mathrm{AU}$ for the inner boundary and 1.24 AU for the outer boundary of the HZ, respectively. Hart [9, 10] calculated the hypothetical evolution of the terrestrial atmosphere over geologic time for different orbital radii. $\mathrm{He}$ found that the HZ, i.e. the "ecological niche" between runaway greenhouse and runaway glaciation processes is amazingly narrow for stars like our Sun: It is delimited from below by $R_{\text {inner }}=0.958 \mathrm{AU}$ and from above by $R_{\text {outer }}=1.004 \mathrm{AU}$, where $R_{\text {inner }} r$ and $R_{\text {outer }}$ are the inner and outer limits to the mean orbital radius, respectively.

A main disadvantage of those calculations was the neglect of the negative feedback between atmospheric $\mathrm{CO}_{2}$ partial pressure and mean global surface temperature via the carbonate-silicate cycle as discovered by Walker et al. [11]. The inclusion of that feedback by Kasting et al. [12] produced the interesting result of an almost constant inner 
boundary of the HZ but a remarkable extension of its outer boundary. In subsequent years, the $\mathrm{HZ}$ approach experienced a number of refinements and the extension to other classes of main sequence stars [13-15]. An overview is provided by Doyle [16]. Recent studies conducted by our group (see particularly, $[17,8]$ ) have generated a rather comprehensive characterization of habitability, based on the possibility of photosynthetic biomass production under large-scale geodynamic conditions. Thus, not only the availability of liquid water on a planetary surface is taken into account but also the suitability of $\mathrm{CO}_{2}$ partial pressure. Our definition of habitability is described in detail in the next part, especially by Eq. (3.5).

At the present time, the determination of habitable zones in extrasolar planetary systems is of special interest, because in the last few years up to $\sim 50$ objects have been identified with the help of novel techniques [18]. Unfortunately, most of the discovered planets are giants on orbits surprisingly close to the central star. Nevertheless, there is hope to find also Earth-like planets with the help of those astronomical observing programs, launched in the early 1990s that rely on planet detection in the Milky Way via gravitational microlensing observation and other techniques [19]. The most

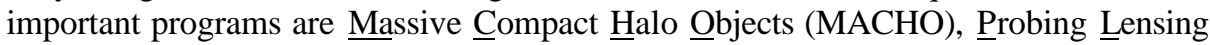
Anomalies Network (PLANET), Experience pour la Recherche d' $\underline{\text { Objects }} \underline{\text { Sombres }}$ (EROS), and Optical Gravitational Lensing Experiments (OGLE).

Gravitational microlensing events occur, when a faint or dark star passes the line of sight of a more distant, brighter star. The light rays emanating from the latter are bent by the gravitational field of the closer, fainter star. This results in a discernible magnification of the image of the brighter object. A planet orbiting the faint star can cause a minor extra peak in the magnification record.

Summarizing we can state that the $\mathrm{HZ}$ is the range of orbital distances from a star, in which a planet can maintain liquid water and biological productivity on its surface. The $\mathrm{HZ}$ can be calculated with the help of climatological approaches or within the framework of Earth system science. According to our model, the HZ for the present solar system extends between about $0.95 \mathrm{AU}$ and about 1.2 $\mathrm{AU}$ [6] and was broader in the past. For extrasolar systems we can postulate a distinct HZ for young central stars in the mass range between about 0.4 and 2 solar masses.

The next two parts describe model calculations for the Sun and for other single main sequence stars, respectively. In the final we give our main conclusions and point out several areas for future work.

\subsection{Models for Calculating the HZ in the Solar System}

Since the early work of Hart [9, 10], there have been many improvements in climatic constraints on the inner and outer boundaries of the HZ. The most comprehensive work in this field is the paper by Kasting et al. [13]. The authors define the boundaries of the $\mathrm{HZ}$ via so-called critical solar fluxes. For the inner radius of the HZ they give three different estimations based on the following assumptions:

1. loss of planetary water by a moist greenhouse [20],

2. loss of planetary water by a runaway greenhouse,

3. observation that there was no liquid water on Venus' surface at least for the last $1 \mathrm{Ga}$. 
The outer radius of the $\mathrm{HZ}$ is also calculated by three different approaches:

1. estimation based on observations and arguments that early Mars had a warm and wet climate (see also the recent papers $[5,21]$ ),

2. maximum possible $\mathrm{CO}_{2}$ greenhouse heating (but see also [22]),

3. first condensation limit of $\mathrm{CO}_{2}$ clouds that increase the planetary albedo.

Assuming the possibility of a "cold start", i.e. an originally ice covered planet that was initially beyond the outer HZ boundary enters the HZ due to HZ boundary shifts with time, Kasting et al. [13] found the following values for the present HZ in the solar system:

1. most conservative case: $0.95 \mathrm{AU} \ldots 1.37 \mathrm{AU}$,

2. least conservative case: $\quad 0.75 \mathrm{AU} \ldots 1.90 \mathrm{AU}$,

3. intermediate case (favored): $0.84 \mathrm{AU} \ldots 1.77 \mathrm{AU}$.

The modeling approach by Franck et al. $[17,8]$ is based on the ideas introduced by Caldeira and Kasting [23]. Therefore, a careful simulation of the coupling between increasing solar luminosity, the silicate rock weathering as parameterized by the mean rate $F_{w r}$, and the global energy balance forms the cornerstone of the investigation. As a direct product, the partial pressure of atmospheric carbon dioxide $P_{a t m}$ and the biological productivity $\Pi$ can be estimated as a function of time $t$ throughout planetary past and future. In the following we give the crucial elements of the causal web employed.

The global energy balance of the planet's climate is usually expressed with the help of the Arrhenius-equation [24].

$$
(1-a) S(t)=4 \sigma T_{b b r}^{4}
$$

where $a$ is the planetary albedo, $\sigma$ is the Stefan-Boltzmann constant, and $T_{b b r}$ is the effective black-body radiation temperature. The time dependence of the solar constant $S$ is fitted with the help of a formula given by Gough [25]. The surface temperature of the planet $T_{s}$ is related to $T_{b b r}$ by the greenhouse warming factor:

$$
T_{s}=T_{b b r}+\Delta T \text {. }
$$

Usually $\Delta T$ is parameterized as a function of $T_{s}$ and $P_{a t m}[23,26]$.

The total process of weathering embraces first the reaction of silicate minerals with carbon dioxide, second the transport of weathering products, and third the deposition of carbonate minerals in sediments. The basic assumptions and limitations of this approach are given in Franck et al. [26]. The weathering rate $F_{w r}$ is a key function in our model. For any given weathering rate the surface temperature $T_{s}$ and the carbon dioxide concentration in the soil $P_{\text {soil }}$ can be calculated self-consistently $[23,26,17]$.

The main role of the biosphere in the context of our model is to increase $P_{\text {soil }}$ in relation to the atmospheric carbon dioxide partial pressure and proportional to the biologic productivity, $\Pi$. $\Pi$ is considered to be a function of temperature and carbon dioxide partial pressure in the atmosphere only.

$$
\frac{\Pi}{\Pi_{\max }}=\left(1-\left(\frac{T_{s}-25^{\circ} \mathrm{C}}{25^{\circ} \mathrm{C}}\right)^{2}\right)\left(\frac{P_{a t m}-P_{\min }}{P_{1 / 2}+\left(P_{a t m}-P_{\min }\right)}\right)
$$


$\Pi_{\max }$ is the maximum productivity and is assumed to be twice the present value [27]. $P_{1 / 2}+P_{\min }$ is the value, at which the pressure dependent factor is equal to $1 / 2$ and $P_{\text {min }}=10 \mathrm{ppm}$ the minimum value for photosynthesis. For fixed $P_{a t m}$, Eq. (3.3) produces maximum productivity at the optimum temperature $\left(T_{s}=25{ }^{\circ} \mathrm{C}\right)$ and zero productivity outside the temperature tolerance interval $\left(0-50^{\circ} \mathrm{C}\right)$.

First we have solved the system of equations under the assumption that the weathering rate $F_{w r}$ is always equal to the present value $F_{w r, 0}$. This is clearly a rather rough approximation. We call this approach the geostatic model (GSM).

Franck et al. [17] have introduced the geodynamic model (GDM). In this case a balance between the carbon dioxide sink in the atmosphere-ocean system and the metamorphic (plate-tectonic) sources is expressed with the help of dimension less quantities [28]:

$$
\mathrm{f}_{\mathrm{wr}} \cdot \mathrm{f}_{\mathrm{A}}=\mathrm{f}_{\mathrm{sr}}
$$

where $f_{w r} \equiv F_{w r} / F_{w r, 0}$ is the weathering rate normalized by the present value, $f_{A} \equiv A_{c} /$ $A_{c, 0}$, is the continental area normalized by the present value, and $f_{s r} \equiv S / S_{0}$ the spreading rate normalized by the present value. With the help of Eq. (3.4) we can calculate the weathering rate from geodynamical theory [17]. Our model is sketched in Fig. 3.1.

Based on our calculation scheme, we define the $\mathrm{HZ}$ as the range of all orbital distances, where the biological productivity is greater than zero:

$$
H Z:=\left\{R \mid \Pi\left(P_{\text {atm }}(R, t), T_{s}(R, t)\right)>0\right\}
$$

In our calculation with the help of the model shown in Fig. 3.1 [17] we found the following values for the present $\mathrm{HZ}: R_{\text {inner }}=0.97 \mathrm{AU}$ and $R_{\text {outer }}=1.39 \mathrm{AU}$.

\subsection{HZ Around Other Main Sequence Stars}

The same type of $\mathrm{HZ}$ calculations, both on the base of climatic constraints and on the base of Earth system modeling as well, can be performed for stars with masses different from the solar mass.

Kasting et al. [13] restricted themselves to stellar lifetimes greater than $2 \mathrm{Ga}$ which corresponds to masses less than $1.5 \mathrm{M}_{\mathrm{s}}\left(1 \mathrm{M}_{\mathrm{s}}=\right.$ one solar mass $)$. At the low-mass end they restricted themselves to masses greater than $0.5 \mathrm{M}_{s}$, because stars with masses $\leq 0.5 \mathrm{M}_{\mathrm{s}}$ show negligible evolution. Stellar luminosities and temperatures were taken directly from Iben $[29,30]$, climatic constraints correspond to their so-called intermediate case. As expected, stellar HZ's for more massive stars are rather short, because they have to be truncated at the end of the main sequence. HZ's for low mass stars are essentially the same over time. In Fig. 3.2 we show the so-called zero age main sequence HZ from Kasting et al. [13] as function of stellar mass.

In our calculation of $\mathrm{HZ}$ in extrasolar planetary systems [8] we used the luminosity evolution of central stars on the main sequence in the mass range between 0.8 and 2.5 $\mathrm{M}_{\mathrm{s}}$. The results have been obtained by polynomial fitting of detailed stellar evolution models like the one presented by Schaller et al. [31]. The temperature tolerance window for the biological productivity given in Eq. (3.3) was extended to the range be- 
tween $0{ }^{\circ} \mathrm{C}$ and $100{ }^{\circ} \mathrm{C}$ in order to incorporate thermophiles [32]. Furthermore, for this study a linear continental growth model was applied. To present the results of our modeling approach, we have delineated the HZ for an Earth-like extrasolar planet at a given but arbitrary distance $R$ in the stellar mass-time plane (Fig. 3.3).

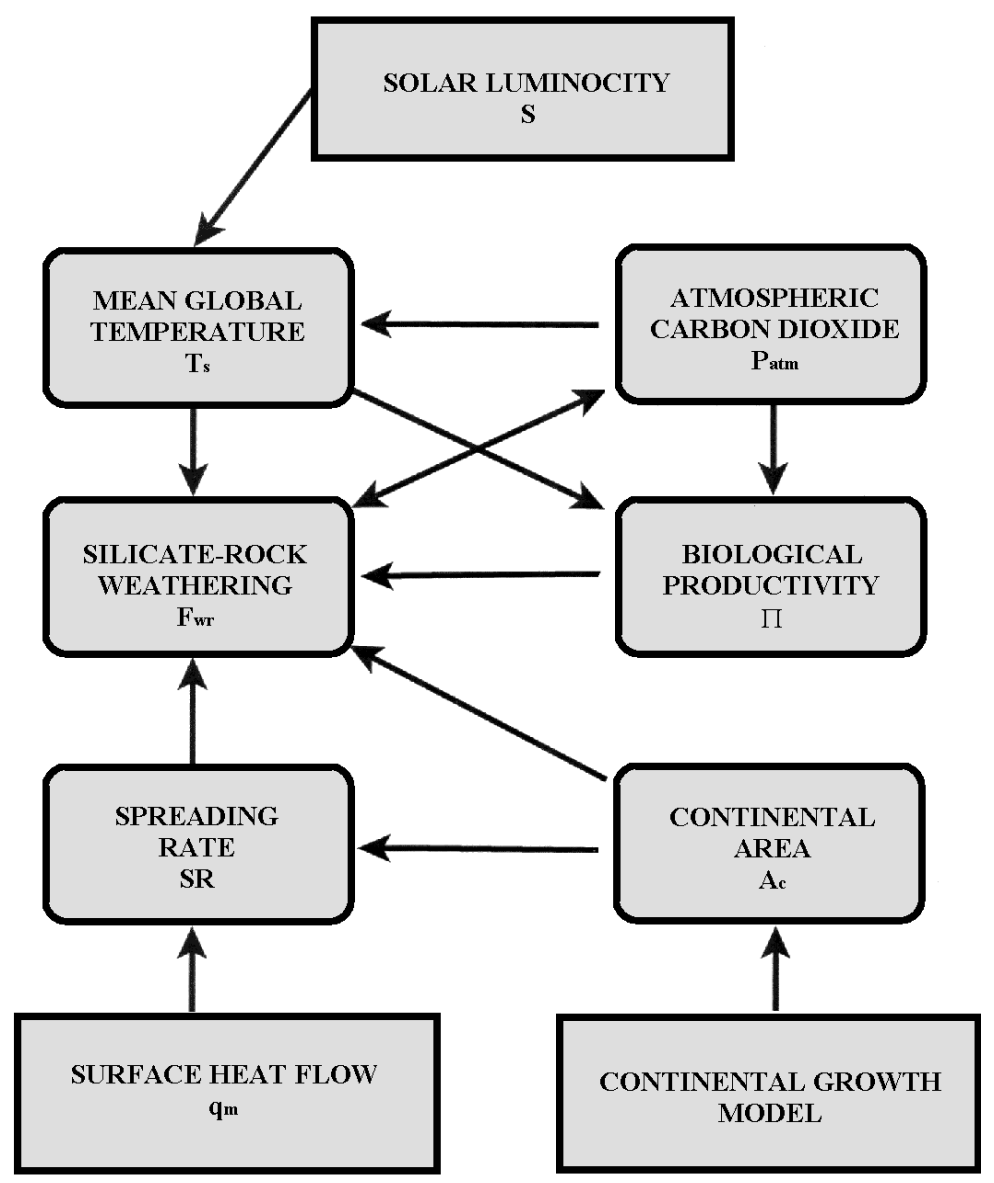

Fig. 3.1 Sketch of our Earth system model. Arrows may describe both negative and positive feedbacks. 


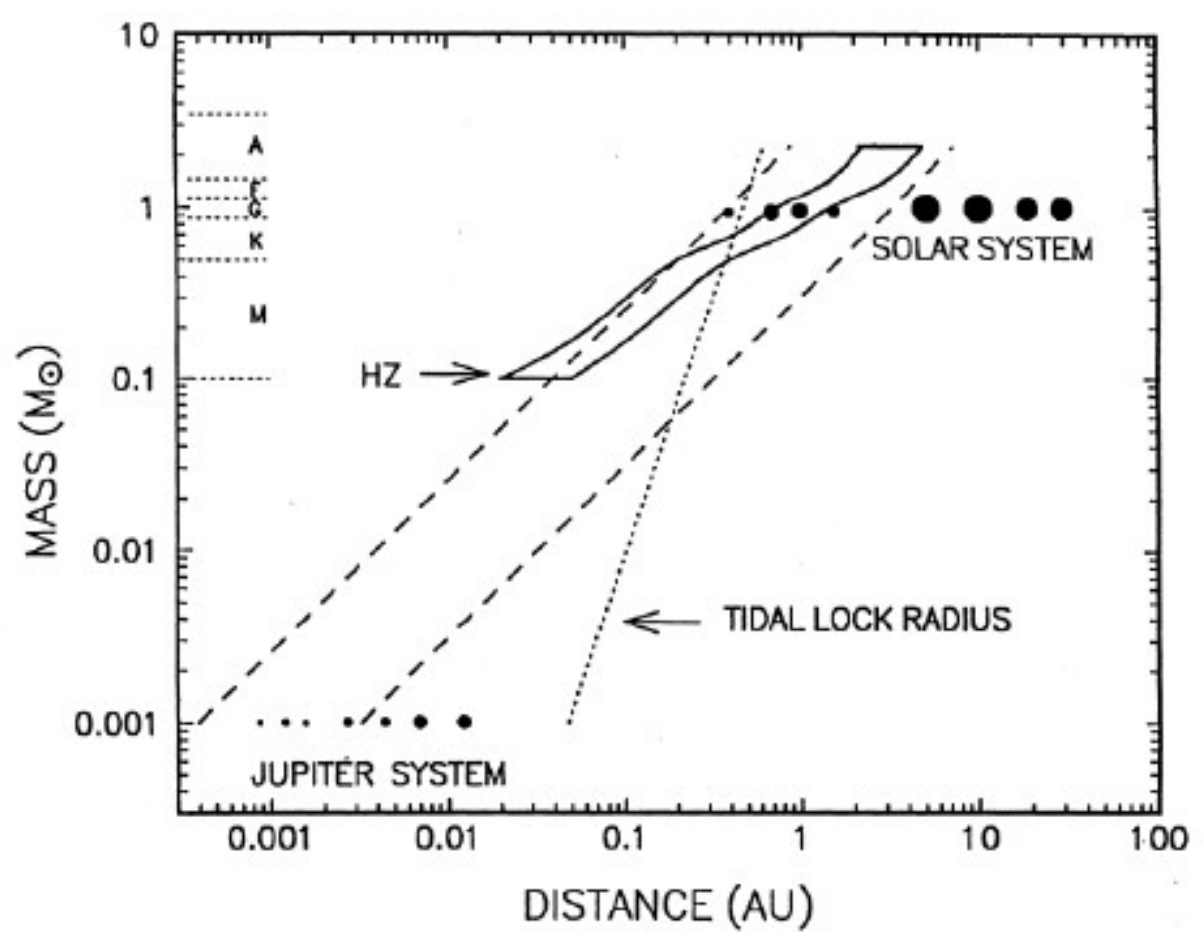

Fig. 3.2 The zero age main sequence $\mathrm{HZ}$ as a function of central star mass (in solar masses $\mathrm{M}_{\odot}$ ) for the intermediate case of climatic constraints. The long-dashed lines delineate the probable terrestrial planet accretion zone. The dotted line is the orbital distance, for which an Earth-like planet in a circular orbit would be locked into synchronous rotation (tidal locking). (Figure of Kasting et al. [13], by kind permission of Dr. J. F. Kasting and Academic Press.)

In Fig. 3.3 the $\mathrm{HZ}$ is limited by the following effects:

1. Stellar lifetime on the main sequence decreases strongly with mass. Using simple scaling laws [33], we estimated the central hydrogen burning period and got $\tau_{\mathrm{H}}<0.8 \mathrm{Ga}$ for $\mathrm{M}>2.2 \mathrm{M}_{\mathrm{s}}$. Therefore, there is no point in considering central stars with masses larger than $2.2 \mathrm{M}_{s}$, because an Earth-like planet may need $\sim 0.8 \mathrm{Ga}$ of habitable conditions for the development of life $[9,10]$. Quite recently, smaller numbers for the time span required for the emergence of life have been discussed, for instance $0.5 \mathrm{Ga}$ [34]. If we perform calculations with $\tau_{\mathrm{H}}<0.5 \mathrm{Ga}$, we obtain qualitatively similar results, but the upper bound of central star masses is shifted to $2.6 \mathrm{M}_{\mathrm{s}}$.

2. When a star leaves the main sequence to turn into a red giant, there clearly remains no HZ for an Earth-like planet. This limitation is relevant for stellar masses in the range between 1.1 and $2.2 \mathrm{M}_{\mathrm{s}}$.

3. In the stellar mass range between 0.6 and $1.1 \mathrm{M}_{\mathrm{s}}$ the maximum life span of the biosphere is determined exclusively by planetary geodynamics, which is inde- 
pendent (in a first approximation, but see limiting effect 4) from $R$. So we obtain the limitation $t<t_{\max }$.

4. There have been discussions about the habitability of tidally locked planets. We take this complication into account and indicate the domain, where an Earthlike planet on a circular orbit experiences tidal locking. That domain consists of the set of $(M, t)$ couples which generate an outer HZ boundary below the tidallocking radius. This limitation is relevant for $M<0.6 \mathrm{M}_{\mathrm{s}}$. As an illustration we depict the HZ for $R=2$ AU in Fig. 3.3.

\subsection{Conclusions}

The question, whether an Earth-like planet discovered outside the solar system may accommodate life, can be answered with the help of Figs. 3.2 and 3.3 if the mass and age of the central star as well as the planet's orbit are known. This is only the present state of the art in theoretical modeling of HZ. There are of course a lot prerequisites for such calculations that have been summarized recently by Lissauer [35].

$\tau_{\mathrm{H}}<0.8 \mathrm{Ga}(\mathrm{I})$

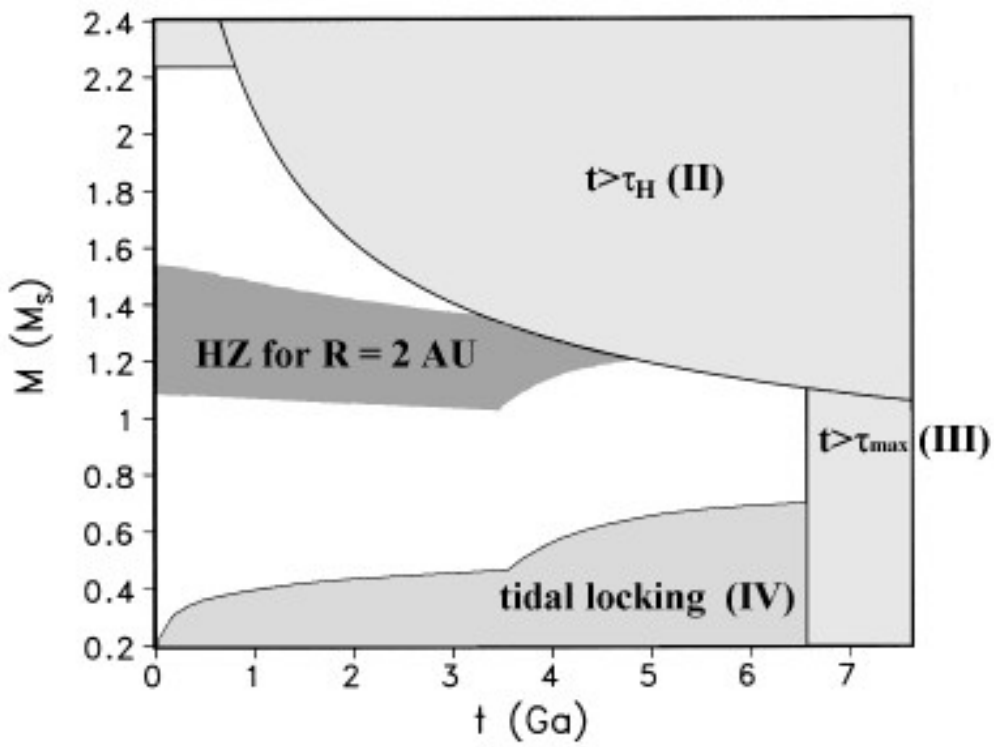

Fig. 3.3 Shape of the HZ (dark grey) in the mass-time plane for an Earth-like planet with photosynthesis at distance $R=2$ AU from the central star. The potential overall domain for accommodating the $\mathrm{HZ}$ of planets at some arbitrary distance is limited by a number of $R$-independent factors that are explained in the text. (Figure slightly changed from Franck et al. [8], copyright by the American Geophysical Union). 
A main assumption is that extraterrestrial life would be carbon-based and needs liquid water. In this way, the carbon-based life is connected with the global carbon cycle between the components of the whole system. The carbon cycle may operate only on a geologically active planet with liquid water. The present understanding of plate tectonics, however, is not sufficient to enable us to predict whether a given planet would exhibit such a phenomenon or not. First theoretical steps to tackle this problem were made by Solomatov and Moresi [36].

Furthermore, planetary orbits are generally chaotic. For stable orbits over a long period of time a minimum separation is required in a system of Earth-like planets [37]. Fortunately, this separation is comparable to the width of the HZ.

To detect Earth-sized extrasolar planets, NASA and ESA are both designing space missions for the second decade of this century. Examples for such project are "Terrestrial Planet Finder" (TPF), i.e. an infrared interferometer operating in an orbit to detect Earth-like extrasolar planets and investigate their atmospheres (http:// astrobiology.arc.nasa.gov), and DARWIN, i.e. an infrared space interferometer to search for signs of life on any Earth-like planet found (http://ast.star.rl.ac.uk/darwin, see Chap. 24, Foing).

Acknowledgements. This work was supported by the German Science Foundation (DFG, grant IIC5-Fr910/9-3) and by the Ministry of Science, Research, and Culture of the State of Brandenburg/Germany (HSP III 1.6; 04/035).

\subsection{References}

$1 \quad$ S.S. Huang, Am. Sci. 47, 397 (1959).

2 S.S. Huang, Sci. Am. 202(4), 55 (1960).

3 S.H. Dole (Ed.) Habitable Planets for Man, Blaisdell, New York, 1964, 158 pp.

4 I.S. Shklovskii, C. Sagan (Eds.) Intelligent Life in the Universe, Holden-Day, San Francisco, 1966, 509 pp.

5 M.P. Golombek, Science 283, 1470 (1999).

6 S. Franck, A. Block, W. von Bloh, C. Bounama, H.J. Schellnhuber, Y. Svrezhev, Planet. Space Sci. 48, 1099 (2000).

7 D.M. Williams, J.F. Kasting, R.A. Wade, Nature 385, 234 (1997).

8 S. Franck, A. Block, W. von Bloh, C. Bounama, M. Steffen, D. Schönberner, H.J. Schellnhuber, JGR-Planets 105, No. E1, 1651 (2000).

9 M.H. Hart, Icarus 33, 23 (1978).

10 M.H. Hart, Icarus 37, 351 (1979).

11 J.C.G. Walker, P.B. Hays, J.F. Kasting, J. Geophys, Res. 86, 9776 (1981).

12 J.F. Kasting, Icarus 74, 472 (1988).

13 J.F. Kasting, D.P. Whitmire, R.T. Reynolds, Icarus 101, 108 (1993).

14 J.F. Kasting, Origins of Life 27, 291 (1997).

15 D.M. Williams, The stability of habitable planetary environments, A Thesis in Astronomy and Astrophysics, Pennsylvania State University, 1998, 140 pp.

16 L.R. Doyle (Ed.), Circumstellar habitable zones, Proc. First International Conference, Travis House Publications, Menlo Park, California, 1996, 525 pp. 
17 S. Franck, A. Block, W. von Bloh, C. Bounama, H.J. Schellnhuber, Y. Svirezhev, Tellus 52B, No.1, 94 (2000).

18 J. Schneider, Extrasolar Planets and Exobiology, http:// www. obspm.fr/encycl/ encycl.html, 2000.

19 D.P. Bennett, S.H. Rhie, Astrophys. J. 472, 660 (1996).

20 J.F. Kasting, O.B. Toon, J.B. Pollack, Sci. Am. 256, 90 (1988).

21 M.C. Malin, K.S. Edgett, Science 288, 2330 (2000).

22 F. Forget, R.T. Pierrehumbert, Science 278, 1273 (1997).

23 K. Caldeira, J.F. Kasting, Nature 360, 721 (1992).

24 S.A. Arrhenius, Philos. Mag. 41, 237 (1896).

25 D.O. Gough, Sol. Phys. 74, 21 (1981).

26 S. Franck, K. Kossacki, C. Bounama, Chem. Geol. 159, 305 (1999).

27 T. Volk, Am. J. Sci. 287, 763 (1987).

28 J.F. Kasting, Am. J. Sci. 284, 1175 (1984).

29 I. Iben, Annu. Rev. Astron. Astrophys. 5, 571 (1967).

30 I. Iben, Astrophys. J. 147, 624 (1967).

31 G. Schaller, D. Schaerer, G. Meynet, A. Meader, Astron. Astrophys. Suppl. Ser. 96-2, 269 (1992).

32 D. Schwartzman, M. McMenamin, T. Volk, Bio Sci. 43, 390 (1993).

33 R. Kippenhahn, A. Weigert (Eds.) Stellar Structure and Evolution, Springer-Verlag, Berlin, 1990, 468 pp.

34 B. Jakosky (Ed.) The Search for Life on Other Planets, Cambridge University Press, Cambridge, 1998, 326 pp.

35 J.J. Lissauer, Nature 402, Supp., C11 (1999).

36 V.S. Solomatov, L.N. Moresi, Geophys. Res. Lett. 24, 1907 (1997).

37 J.J. Lissauer, Rev. Mod. Phys. 71, 835 (1999). 